\title{
Modulation of Dynamics and Reactivity of Water in Reverse Micelles of Mixed Surfactants
}

\author{
Rajib Kumar Mitra, Sudarson Sekhar Sinha, Pramod Kumar Verma, and Samir Kumar Pal* \\ Unit for Nano Science \& Technology, Department of Chemical, Biological \& Macromolecular Sciences, S.N. \\ Bose National Center for Basic Sciences, Block JD, Sector III, Salt Lake, Kolkata 700098, INDIA
}

Received: April 24, 2008; Revised Manuscript Received: July 21, 2008

\begin{abstract}
In this contribution, we attempt to correlate the change in water dynamics in a reverse micellar (RM) core caused by the modification of the interface by mixing an anionic surfactant, sodium bis(2-ethylhexyl) sulfosuccinate (AOT), and a nonionic surfactant, tetraethylene glycol monododecyl ether (Brij-30), at different proportions, and its consequent effect on the reactivity of water, measured by monitoring the solvolysis reaction of benzoyl chloride $(\mathrm{BzCl})$. The dimension of the RM droplets at different mixing ratios of AOT and Brij-30 $\left(\mathrm{X}_{\mathrm{Brij}-30}\right)$ has been measured using dynamic light scattering (DLS) technique. The physical properties of the RM water have been determined using Fourier transform infrared spectroscopy (FTIR) and compressibility studies, which show that with increasing $\mathrm{X}_{\mathrm{Brij}-30}$, the water properties tend toward that of bulk-like water. The solvation dynamics, probed by coumarin 500 dye, gets faster with $\mathrm{X}_{\text {Brij-30. The rotational anisotropy studies }}$ along with a wobbling-in-cone analysis show that the probe experiences less restriction at higher $\mathrm{X}_{\mathrm{Brij}-30}$. The kinetics of the water-mediated solvolysis also gets faster with $\mathrm{X}_{\mathrm{Brij}-30}$. The increased rate of solvolysis has been correlated with the accelerated solvation dynamics, which is another consequence of surfactant headgroupwater interaction.
\end{abstract}

\section{Introduction}

Reverse micelles (RMs) provide local hydrophilic moiety in an organic phase, which is an attractive model system for biomolecules since they can mimic several important and essential features of biological membranes. The entrapped water inside the RM core has properties interestingly different from those in the bulk. ${ }^{1,2}$ This unique feature of entrapped water inside the RM can be exploited fruitfully in various applications in the field of biotechnology. The physicochemical properties of the entrapped water has been found to be strongly dependent on the chemical nature of the dispersant phase (oil), surfactant and also on the hydration level of the RM $\left(w_{0}=\right.$ [water]/ [surfactant]). Solubilization of biological macromolecules (proteins, DNA, enzymes) in RM has recently gained much importance. ${ }^{3-10}$ The three governing factors for solubilization are electrostatic, hydrophobic and interactions of the RM interface with specific moiety or segments of solubilized biological macromolecules (proteins, enzymes, DNA etc.). The most widely studied RM for protein encapsulation is that made by the anionic surfactant sodium bis(2-ethylhexyl) sulfosuccinate (AOT) as it can form spherical RMs over wide range of $w_{0}$ values. ${ }^{11,12}$ In this system, the protein uptake is governed by the electrostatic interaction between AOT anion and the positive charge of the protein at a $\mathrm{pH}$ lower than the isoelectric point. However, difficulties arise for proteins that are solubilized in the RM by hydrophobic interactions. Also electrostatic interaction can compete with the biospecific interaction to disturb the high selectivity in the extraction of proteins. A unique remedy of this problem is to dope the interface with nonionic surfactants, since they have weaker interaction with proteins compared to the ionic surfactants. The most commonly used nonionic surfactant is the poly(oxyethylene) alkyl ether $\left(C_{i} E_{j}\right)$ family in which the hydrophilic-lipophilic balance (HLB) ${ }^{13}$ can easily be tuned by changing $i$ and $j$. Modification of the interface by

* Corresponding author. E-mail: skpal@bose.res.in. mixing the surfactants produces considerable changes in the elastic rigidity of the interface which in turn affects the water solubilization capacity and microvisocosity of entrapped water of the RMs depending upon the extent of penetration of the nonionic surfactant in the interphase. But whether such a modification of the interface produces considerable effects on the dynamics of the confined water is a question yet to be addressed. Previous studies of RM systems involving more than one surfactant using solubilization, ${ }^{14-19}$ conductivity, ${ }^{20-24}$ viscosity, ${ }^{25}$ spectroscopic, ${ }^{26-28}$ SANS,${ }^{29-31}$ FTIR and NMR measurements ${ }^{32}$ identify significant modification of the interface and water structure in RM systems. However, the effect of mixing of surfactants on the dynamics of water has never been explored. It has recently been reported that addition of nonionic surfactant into the interface of ionic surfactants produces significant modification of enzyme activity in the RM core, ${ }^{33-35}$ polymer synthesis ${ }^{36}$ nanoparticle synthesis ${ }^{37}$ etc. However, a proper understanding of the process involved is still lacking. The probable reasons might be either the change in the reactivity of the entrapped water or the modified interaction of the biomolecules with the interface or both. It is well-known that the dynamics of water molecules inside RM is highly restricted. A recent study ${ }^{38}$ has shown that the confinement of water in the $\mathrm{RM}$ interior is responsible for the observed slow relaxation dynamics, irrespective of the charge of the interface. But still significant differences have been observed between the dynamical nature of water in RM containing ionic surfactant and nonionic surfactant. Levinger et al. ${ }^{39-41}$ reported the ultrafast solvation dynamics of both ionic (AOT) and nonionic (Brij30) RM systems and compared the results to find that solvation dynamics of the former is slower than that of the latter. A possible source for the observed difference might be the difference in the interaction of water with the charged and uncharged interface. An interesting question that emerges from these reports is whether one can tune the dynamics of confined water in RM by mixing the two types of surfactants. It is known 
that by varying the hydration level of RM one can easily tune the dynamics, but the additional advantage of mixing the surfactants lies in the fact that encapsulated biomolecules would experience less interaction with the interface, and it might be possible to tune the rate of chemical reaction in RM core keeping the water content constant. Another interesting question is that if one tunes the dynamics of the entrapped water by mixing surfactants, would that also modify the reaction affinity (nucleophilicity or electrophilicity) of it. Very recently Singh et al. ${ }^{42}$ has reported that in a surfactant-polymer supramolecular assembly (mixed micelle), the chemical reactivity of a reactant dissolved in it can be tuned substantially by changing the composition of the supramolecular assembly. However, to the best of our knowledge no group has ever attempted to correlate the dynamics and reactivity of entrapped water in RM consisting of mixed surfactant systems.

In the present contribution we have tried to understand the change in solvation dynamics of water in RMs constituted of an anionic surfactant (AOT) and a nonionic surfactant (Brij30 ) in isooctane with different mixing ratios using time-resolved fluorescence spectroscopy probed by coumarin 500 (C500). Brij30 (commercial analogue to $\mathrm{C}_{12} \mathrm{E}_{4}$ ) is a well studied nonionic surfactant ${ }^{43-45}$ that can form RM without the addition of any cosurfactant. The choice of the probe is based on the fact that when excited at $409 \mathrm{~nm}$, only the probes residing at the interface and/or facing the polar core get excited. ${ }^{46,47}$ Thus the spectroscopic information could be assumed to be responses from the interfacial region only. Since our study is focused on the change in the dynamics of water with the modification of interface, the choice of the probe finds its validity. To understand the geometrical restriction of the probe at the interface, rotational relaxation dynamics of the dye in different RM systems have also been determined. Phase mapping the oil-rich zone of the ternary system has been carried out and the dynamics has been measured for the $w_{0}=2.5$ system with different mixing ratios of AOT and Brij-30. The micellar sizes at different mixing ratios have been measured by dynamic light scattering (DLS) technique. The physicochemical characteristic of the entrapped water has been determined using Fourier transform infrared spectroscopy (FTIR) and compressibility measurements. Finally, to correlate the dynamics with reactivity, we measure the kinetics of solvolysis of benzoyl chloride $(\mathrm{BzCl})$ in the RMs of different surfactant composition.

\section{Materials and Methods}

Sodium bis(2-ethylhexyl) sulfosuccinate (AOT) and tetraethylene glycol monododecyl ether (Brij-30) [Scheme 1] were products of Sigma and isooctane $(i-\mathrm{Oc})$ was a product of Spectrochem. Benzoyl chloride $(\mathrm{BzCl})$ was purchased from Merck. All the chemicals were used without further purification. Coumarin-500 (C-500) [Scheme 1] was obtained from Exciton. AOT and Brij-30 were dissolved in $i$-Oc at a concentration of $200 \mathrm{mM}$ to prepare two stock solutions and then mixed in desired proportions. The mole fraction of Brij-30, $\mathrm{X}_{\mathrm{Brij}-30}=$ $[$ Brij-30]/([AOT] $+[$ Brij-30]) was varied from 0 to 1 . Calculated amount of water was injected into it to produce the reverse micelles $(\mathrm{RMs})$ of $w_{0}=2.5$. Steady-state absorption and emission were measured with Shimadzu UV-2450 spectrophotometer and Jobin Yvon Fluoromax-3 fluorimeter respectively. The kinetics of solvolysis of $\mathrm{BzCl}$ was measured in Shimadzu UV-2450 spectrophotometer by monitoring the time dependent change in the absorbance of $\mathrm{BzCl}$ at $288 \mathrm{~nm}$. To determine the phase behavior, water is added in small volumes to a surfactant-oil mixture. The initial concentration of the

\section{SCHEME 1: Molecular Structure of Coumarin 500,} AOT, and Brij-30

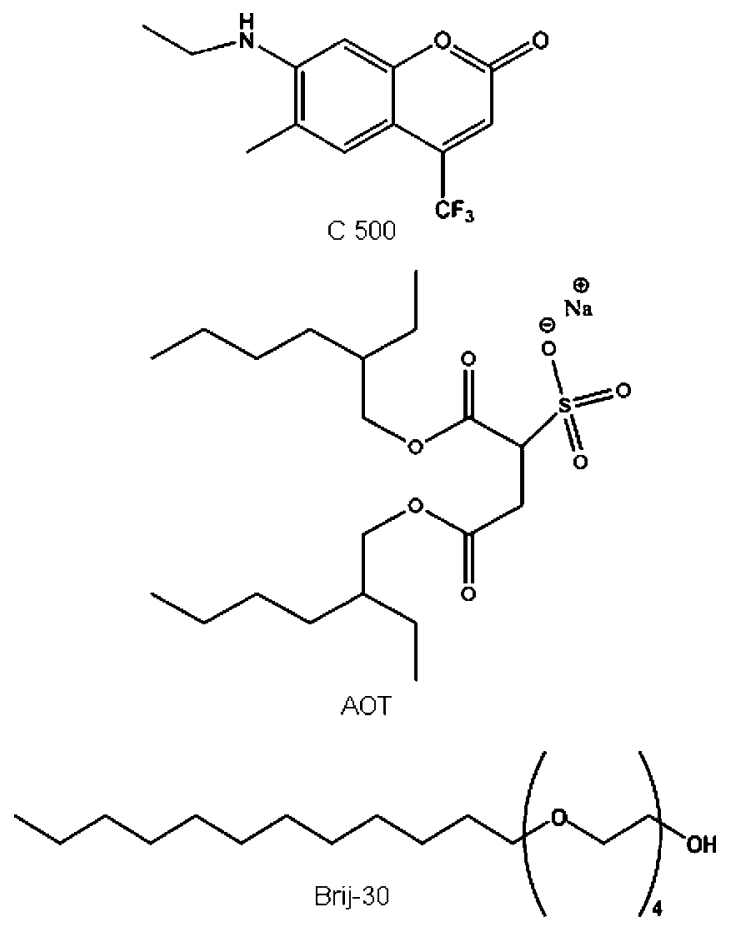

surfactant(s) in oil was fixed at $200 \mathrm{mM}$. After each addition of water, the system was shaken vigorously in a vortex shaker and kept for at least $5 \mathrm{~min}$ in a water bath. The transparency of the system is checked visually. The phase boundary contains an uncertainty of $\pm 10 \%$. DLS measurements were carried out with Nano-S Malvern-instrument employing a $4 \mathrm{~mW} \mathrm{He}-\mathrm{Ne}$ laser $(\lambda=632.8 \mathrm{~nm})$ equipped with a thermostated sample chamber. All the scattered photons were collected at $173^{\circ}$ scattering angle. The scattering intensity data were processed using the instrumental software to obtain the hydrodynamic diameter $\left(d_{H}\right)$ and the size distribution of the scatterer in each sample. The instrument measures time dependent fluctuation in the intensity of light scattered from the particles in solution at a fixed scattering angle. Hydrodynamic diameter $\left(d_{H}\right)$ of the micelles was estimated from the intensity autocorrelation function of the time-dependent fluctuation in intensity. $d_{H}$ is defined as

$$
d_{H}=\frac{k_{b} T}{3 \pi \eta D}
$$

where $k_{\mathrm{b}}$ is the Boltzmann constant, $\eta$ is the viscosity, and $D$ is the translational diffusion coefficient. FTIR spectra were recorded on a JASCO FTIR-6300 spectrometer (absorption mode) using $\mathrm{CaF}_{2}$ window.

Volume and compressibility of the mixtures have been calculated using the density and sound velocity values measured by a density meter; model DSA5000 from Anton Paar (Austria) with an accuracy of $5 \times 10^{-6} \mathrm{~g} \mathrm{cc}^{-1}$ and $0.5 \mathrm{~ms}^{-1}$ in density and sound velocity measurements, respectively. The calculations for volume and compressibility determinations are made in the following manner ${ }^{48-50}$ adiabatic compressibility $(\beta)$ of the mixture can be determined by measuring the solution density $(\rho)$ and the sound velocity $(u)$ and applying the Laplace's equation 


$$
\beta=\frac{1}{\rho u^{2}}
$$

Adiabatic compressibility of water in the present study is calculated to be $4.47 \times 10^{-10} \mathrm{~Pa}^{-1}$, which is close to the literature value of $4.5 \times 10^{-10} \mathrm{~Pa}^{-1}$ for bulk water. The apparent specific volume of the solute (water), $\phi_{v}$, is given by

$$
\phi_{v}=\frac{1}{\rho_{0}}+\frac{\rho_{0}-\rho}{c \rho_{0}}
$$

where $\mathrm{c}$ is the concentration of water, $\rho_{0}$ and $\rho$ are the densities of the dry RM $\left(w_{0}=0\right)$ and $\mathrm{RM}$ at $w_{0}=2.5$ respectively. The partial apparent adiabatic compressibility $\left(\phi_{k}\right)$ of water is obtained from the following relation

$$
\phi_{k}=\beta_{0}\left(2 \phi_{v}-2[u]-\frac{1}{\rho_{0}}\right)
$$

where $[u]$ is the relative specific sound velocity increment given by,

$$
[u]=\frac{u-u_{0}}{u_{0} c}
$$

where $\mathrm{u}_{0}$ and $\mathrm{u}$ are the sound velocities in dry RM $\left(w_{0}=0\right)$ and $\mathrm{RM}$ at $w_{0}=2.5$, respectively.

Fluorescence transients were measured and fitted by using commercially available spectrophotometer (LifeSpec-ps) from Edinburgh Instrument, U.K. (excitation wavelength 409 nm, 80 ps instrument response function (IRF)). The details of timeresolved measurements can be found elsewhere. ${ }^{47,51}$ The time dependent fluorescence Stokes shifts, as estimated from TRES, were used to construct the normalized spectral shift correlation function or the solvent correlation function $C(t)$ defined as

$$
C(t)=\frac{v(t)-v(\infty)}{v(0)-v(\infty)}
$$

where, $v(0), v(t)$ and $v(\infty)$ are the emission maximum (in $\mathrm{cm}^{-1}$ ) at time zero, $t$, and infinity, respectively. The $v(\infty)$ values had been taken to be the emission frequency beyond which an insignificant or no spectral shift was observed. The $C(t)$ function represents the temporal response of the solvent relaxation process, as occurs around the probe following its photo excitation and the associated change in the dipole moment. For anisotropy $(r(t))$ measurements, emission polarization was adjusted to be parallel or perpendicular to that of the excitation and anisotropy is defined as

$$
r(t)=\frac{\left[I_{\text {para }}(t)-G \times I_{\text {perp }}(t)\right]}{\left[I_{\text {para }}(t)+2 \times G \times I_{\text {perp }}(t)\right]}
$$

$G$, the grating factor was determined following longtime tail matching technique. ${ }^{52}$ All the anisotropies were measured at the emission maxima.

\section{Results and Discussion}

For a pseudoternary RM system consisting of mixed surfactant, it is important to have an idea of the phase diagram of the system. We construct the phase diagram of the AOT/Brij-30/ $i$-Oc/water system in the oil-rich region as a function of $w_{0}$ and is depicted in Figure 1. In this system the single-phase region can be assumed to have an $\mathrm{L}_{2}$ configuration (water-in-oil microemulsion). It can be observed that AOT/i-Oc RM ( $X_{\text {Brij-30 }}$ $=0$ ) can solubilize water up to $w_{0} \sim 22$ whereafter a cloudy region (optically not transparent) occurs. On further addition of water, the system becomes clear at $w_{0} \sim 30$ and can solubilize

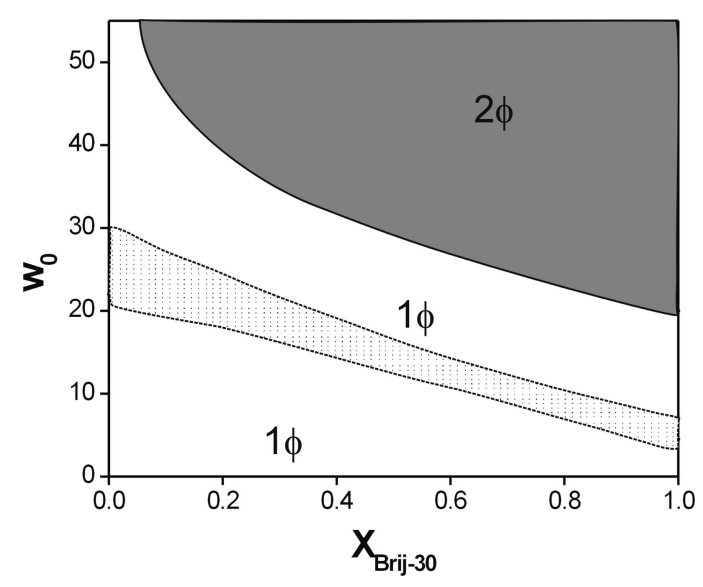

Figure 1. Phase diagram of the system AOT/Brij-30/isooctane/water at $25{ }^{\circ} \mathrm{C}$ with different mixing proportions of AOT and Brij-30. $1 \phi$ and $2 \phi$ indicate single-phase and biphasic systems, respectively. The dotted region lying between the two $1 \phi$ regions is the cloudy region.

water up to $w_{0} \sim 65$. This cloudy region consists of several mesophases like, viscous and gel-like phases. Such a phase behavior is not very often encountered in the literature. Appearance of a such a cloudy region in between two monophases has previously been reported for the AOT system [AOT/1-butanol/eucalyptus oil/water ${ }^{53}$ (see Figure 2 of the reference)]. Due to its cone like shape, AOT is one of the most suitable surfactants to form RM with. However, depending upon the content of water, the initial hydration of the headgroup region may vary, which can induce the formation of different mesophases like liquid crystalline phase, lamellar phase, bicontinuous phase etc. ${ }^{54,55}$ Addition of further water then gradually produces water-in-oil microemulsions, and when a solubilization limit is reached, the RM droplet coalesce to extract the excess water into a separate phase (formation of the biphasic region). For the nonionic surfactant $\mathrm{C}_{12} \mathrm{E}_{4}$, a similar observation has earlier been reported by Merdas et al. ${ }^{56}\left(\mathrm{C}_{12} \mathrm{E}_{4} /\right.$ dodecane/water $\mathrm{RM}$ systems, see Figure 1 of the reference). They identified the two isotropic phases as the water poor and water rich phases. Since our main objective of the phase study is only to ascertain the water solubilization capacity of mixed RM to get monophasic systems, no attempt has been made to make an elaborate study to identify the exact boundary of such mesophases. As the interface is progressively doped with Brij-30, the solubilization capacity decreases. Such a decrease in solubilization capacity with increasing Brij-30 content has previously been reported by Chatterjee et al. ${ }^{27}$ The solubilization capacity of RM systems depends upon various parameters like oil, temperature, ionic strength of the aqueous phase, interfacial composition etc. ${ }^{17,18,57}$ In the present system, keeping other parameters constant, the

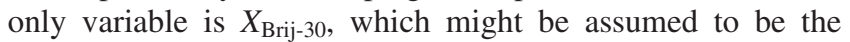
governing factor of the significant alteration of solubilization capacity. The decreased solubilization capacity of the nonionic system is indicative of greater interaction between the micelles, which could arise due to the increased chain length of nonionic surfactant resulting in an enhancement of the droplet coagulation followed by phase separation. A decrease in the conductance percolation threshold for the AOT/Brij-30 mixed RM system compared to the AOT system ${ }^{27}$ supports the droplet coagulation at smaller water content. The penetration of nonionic surfactant in the interface plays a decisive role on the fluidity of the interface. ${ }^{21}$ If the nonionic surfactant headgroup is less polar, it can penetrate less efficiently into the aqueous phase and would interact less efficiently with the entrapped water. This fact 

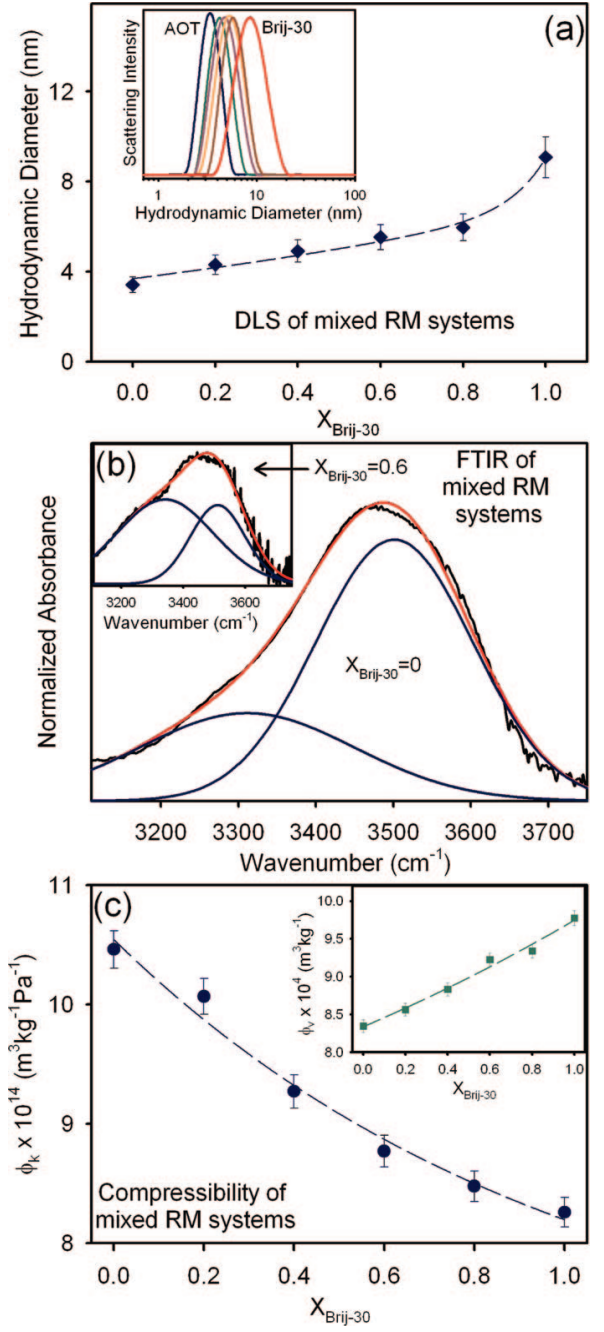

Figure 2. (a) Hydrodynamic diameter of AOT/Brij-30/isooctane/water mixed reverse micellar systems at $\mathrm{w}_{0}=2.5$ as a function of $X_{\mathrm{Brij}-30}$. The broken line is a guide to the eye. Typical DLS signals for all the systems are shown in the inset. (b) FTIR spectra of AOT/isooctane/water at $w_{0}$ $=2.5$. The black curve is the experimental one, red curve is the overall fitted curve and the blue curves are the deconvoluted curves. A similar spectra for the mixed system at $X_{\mathrm{Brij}-30}=0.6$ is shown in the inset. (c) Compressibility data for the AOT/Brij-30/isooctane/water mixed reverse micellar systems at $w_{0}=2.5$ as a function of $X_{\mathrm{Brij}-30}$. The broken lines are guide to the eye.

corroborates well with the present finding that solubilization capacity of mixed RM decreases with increasing $X_{\mathrm{Brij}-30}$. It is evident from figure 1 that Brij-30/isooctane system can form $\mathrm{RM}$ up to $w_{0} \sim 20$, but in the $w_{0}=5-10$ region, it fails to form isotropic solution and a DLS measurement in this region produces inconsistent result of size distribution pointing out to the instability of the system. Such phase behavior restricts us from choosing any $w_{0}$ value (up to 20, as it is the maximum solubilization limit of Brij-30/isooctane system) for the study and it is found that $w_{0}=2.5$ is the most suitable one as all the mixed systems form clear monophasic dispersions. It could also be noted that for $\mathrm{C}_{12} \mathrm{E}_{4} /$ hydrocarbon systems, the shape of RMs deviate from spherical beyond $w_{0}=4{ }^{43}$ Such a small value of $w_{0}$ has an additional advantage in the present system. As $w_{0}$ increases, the waterpool size in a RM and consequently the extent of bulk-like water increases and the solvation dynamics of a probe in such systems become faster ${ }^{41}$ For such ultrafast process our picosecond-resolved TCSPC system does not resolve a large portion of the dynamical Stokes shift and it would have been difficult to compare the change in the Stokes shift for different mixed systems.

Figure 2 depicts the characterization of the mixed RM systems using different techniques. The sizes of the RMs are determined using the DLS measurements (Figure 2a). It can be observed that AOT/i-Oc system produces spherical and monodispersed droplets with hydrodynamic diameter of $3.4 \mathrm{~nm}$. SAXS measurements confirms the water pool radius of AOT/i-Oc RM system of $\sim 0.9 \mathrm{~nm}$ at $w_{0}=3,{ }^{58}$ and assuming the AOT chain length to be $\sim 1 \mathrm{~nm}$, the observed hydrodynamic diameter in the present study is in good agreement with the previous results. As $X_{\mathrm{Brij}-30}$ is increased, the hydrodynamic diameter of the droplet increases. Such an increase in the droplet size with increasing Brij-30 content has previously been reported by Liu et al. ${ }^{20}$ It could be noted that Brij-30 has a longer tail length of 12 carbon atoms compared to the 8 carbon atoms in AOT. A molecular dynamics simulation study involving Brij-30 RMs ${ }^{44}$ shows that the carbon atoms can form "all-trans" or "helix" conformation, which can in turn induce ellipticity in the droplet configuration. The overall increase in the hydrodynamic diameter might reflect the extended tail part of the micelles as well as an increase in the water core radius of the micelles. Pant et al ${ }^{39}$ previously showed that the radius of Brij-30/cyclohexane RM system at $w_{0}=2.5$ is $2.6 \mathrm{~nm}$. Assuming $i$-Oc to be a less penetrating oil molecule compared to cyclohexane, ${ }^{18}$ a larger droplet size could be assumed. MD simulation shows that for $w_{0}=3$, Brij-30 produces a water core radius of $1.5-1.9 \mathrm{~nm}$. Time resolved fluorescence quenching studies by Vasilescu et al ${ }^{43}$ identified a polar core radius of $2-3 \mathrm{~nm}$ for $\mathrm{C}_{12} \mathrm{E}_{4} / \mathrm{dec}$ ane system in the $w_{0}$ range of 1.3-4.0. The present DLS study shows a slow increase in the hydrodynamic diameter of the droplets up to $X_{\text {Brij-30 }}=0.8$, and assuming the hydrophobic tail of Brij-30 to contribute significantly in the increased size, the water core radius can be assumed to increase marginally with $X_{\text {Brij-30 }}$. However, for the Brij-30 system $\left(X_{\mathrm{Brij}-30}=1.0\right)$, the large increase in the droplet size can be due to the deformed shape of the droplets (as has been observed for the Brij-30/dodecane system $^{43,56}$ ) as well as increased hydration of the surfactant headgroup.

To understand the physical nature of water molecules entrapped in the RM core, we carry out FTIR measurements to determine the $\mathrm{O}-\mathrm{H}$ stretching frequency in different mixed $\mathrm{RM}$ systems. To get rid of the $\mathrm{C}-\mathrm{H}$ stretching frequency, the observed spectra at $w_{0}=0$ has been subtracted from the $w_{0}=$ 2.5 spectra, and the differential spectra have been analyzed. A representative result is depicted in Figure 2b. It is known that different types of hydrogen bonded water molecules exist in $\mathrm{RM},{ }^{59,60}$ although there are reports ${ }^{61-63}$ that point out that these water molecules can broadly be classified into two major classes, namely headgroup bound and bulk-like water molecules. The differential IR spectra obtained in the present study have been deconvoluted into two peaking at $\sim 3500$ and $\sim 3300 \mathrm{~cm}^{-1}$, corresponding to the $\mathrm{O}-\mathrm{H}$ stretching frequency of the headgroup bound and bulk-like water, respectively. For the AOT/ $i$-Oc RM system, it can be observed that the area under the $3500 \mathrm{~cm}^{-1}$ curve is larger than the corresponding $3300 \mathrm{~cm}^{-1}$ curve, reflecting that the fraction of bound water molecules is higher than that of the bulk-like water molecules. At such a low level of hydration most of the water molecules solvate the polar headgroup of AOT. With increasing $X_{\mathrm{Brij}-30}$, the peak at $3300 \mathrm{~cm}^{-1}$ strengthens at an expense of the $3500 \mathrm{~cm}^{-1}$ peak. A representative figure for the $X_{\mathrm{Brij}-30}=0.6$ has been shown in the inset of Figure 2b. It is evident that almost equal amount of both type of water exists in this mixed RM. As the amount of 
water is fixed (fixed $w_{0}$ ), it can be assumed that the water molecules are interacting less strongly with the nonpolar headgroup in comparison to the AOT headgroup. In a previous study, ${ }^{64}$ using nonylphenyl polyoxyethylene (IGEPAL) surfactant RMs in cyclohexane, the FTIR spectra in the 3100-3700 $\mathrm{cm}^{-1}$ range was deconvoluted into three curves peaking at 3542, 3425 , and $3329 \mathrm{~cm}^{-1}$, with the relative contribution of the red peak to be the most prominent one indicating the presence of a large fraction of bulk like water in the RMs. Although the nonionic surfactant used in the present study structurally differ from that of IGEPALs due to the presence of phenyl ring in the latter, but the overall feature of the FTIR spectra of the former corroborates well with the present study.

To further support this observation, we perform the compressibility study of the mixed RM systems. The results are shown in Figure $2 \mathrm{c}$ and a table containing all the measurements can be found in the Supporting Information section (Table S1). It can be observed that the apparent specific volume of water is low in the AOT/i-Oc RM and increases regularly with increase in $X_{\mathrm{Brij}-30}$ and reaches a bulk-like value of $1.003 \times 10^{-3} \mathrm{~m}^{3} \mathrm{~kg}^{-1}$. A similar increase in $\phi_{v}$ has previously been reported for both AOT and Brij-30 RM with increase in the water content. ${ }^{50}$ The increase in the $\phi_{v}$ with increasing $X_{\mathrm{Brij}-30}$ indicates more intermolecular hydrogen bond formation between water molecules and poor interaction between Brij head groups and water molecules as the system reaches a bulk-like behavior. On the other hand, the apparent specific adiabatic compressibility $\left(\phi_{k}\right)$ decreases regularly with increasing $X_{\mathrm{Brij}-30}$. The $\phi_{k}$ values obtained in the present study for the single surfactant systems are in good agreement with previously reported results. ${ }^{50} \mathrm{It}$ could be noted that the $\phi_{k}$ values for the AOT system are higher in magnitude than the Brij-30 systems. The decrease in $\phi_{k}$ with $X_{\text {Brij-30 }}$ indicates that the water molecules tend toward a bulklike behavior. This observation supports the results obtained from FTIR measurements that Brij-30 headgroup interacts less strongly with the water molecules as does the AOT headgroup, thereby increasing the ratio of bulk-like to bound type water molecules.

The above discussion confirms that the change in interfacial composition affects the physical properties of water significantly. Now we investigate how this change affects the dynamics of the trapped water molecules. In earlier studies, the probe C500 has been demonstrated to be a potential reporter of the solvation dynamics in AOT/i-Oc system. ${ }^{46,47}$ Here, we study the solvation dynamics of the probe in the mixed RM systems with varying

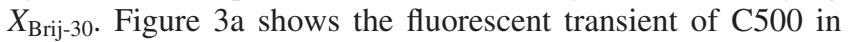
AOT/RM at three different wavelengths of 420, 490, and 590 $\mathrm{nm}$. It could be noted that the transient at the blue end is associated with decay components of $0.2(51 \%), 1.2(27 \%)$, and $3.4 \mathrm{~ns}(22 \%)$, whereas the transient at the red end is characterized with the presence of a significant rise component of $1.5 \mathrm{~ns}$ along with a decay component of $5.8 \mathrm{~ns}$. Such a phenomenon is a clear indication of solvation of the probe molecule. As evidenced from the figure, the emission spectrum suffers a consistent red shift with time corresponding to a significant amount of dynamical Stokes shift (Table 1). We construct the solvent correlation function, $C(t)$ according to eq 6 and two representative curves are shown in Figure $3 \mathrm{~b}$. All the curves are fitted biexponentially and the results are presented in table 1 . Some of the curves provide with better $\chi^{2}$-square values when fitted with triexponential functions, but the average solvation time $\left\langle\tau_{s}\right\rangle$, expressed as $\left\langle\tau_{s}\right\rangle=\sum_{i} a_{i} \tau_{i}$ (where $a_{i}$ is the preexponential factor and $\tau_{i}$ is the time constant), does not change appreciably by a triexponential fitting. Thus we prefer to fit the
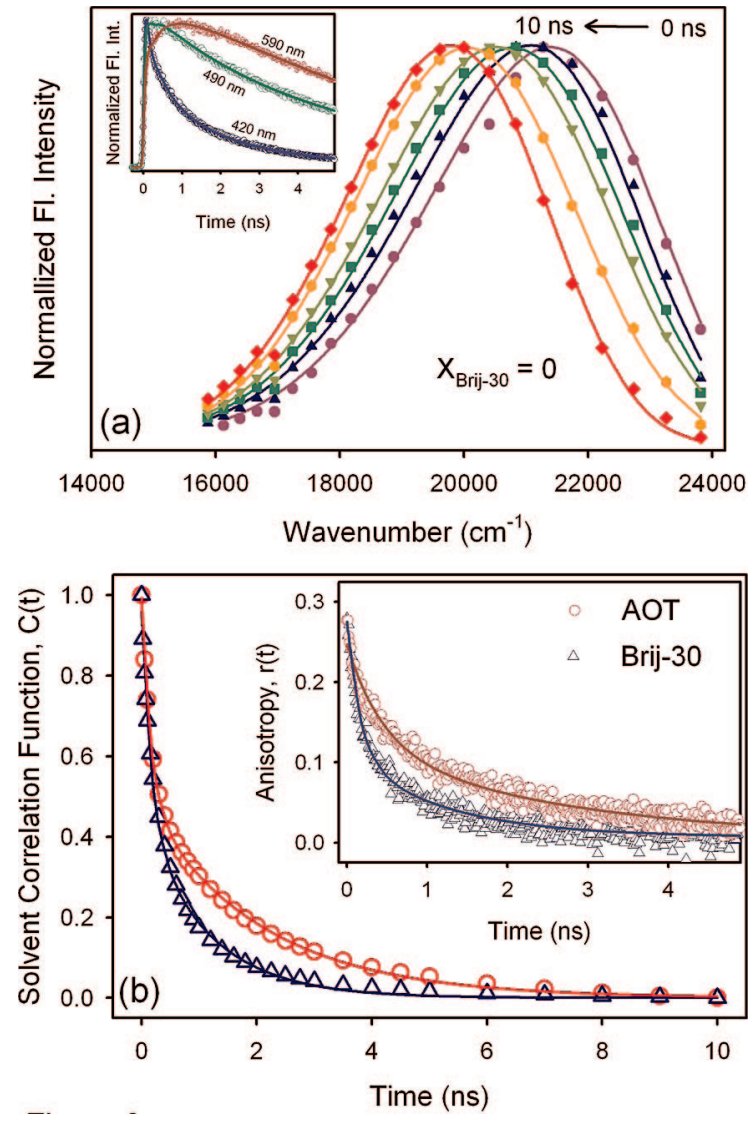

Figure 3. (a) TRES of C500 in AOT/isooctane/water mixed reverse micelle at $\mathrm{w}_{0}=2.5$. The transients at 420,490 and $590 \mathrm{~nm}$ are shown in the inset. (b) Solvent correlation function of AOT/isooctane/water and Brij-30/isooctane/water reverse micelles at $\mathrm{w}_{0}=2.5$. The corresponding time-resolved anisotropies are shown in the inset. The solid lines are the best fitted curves.

TABLE 1: Solvation Dynamics of C500 and Reaction Kinetics of Solvolysis of Benzoyl Chloride in AOT/Brij-30/ Isooctane/Water Mixed Surfactant Reverse Micellar Systems at $w_{0}=2.5$

\begin{tabular}{lccccccc}
\hline & & & & & & & $K_{\text {obs }} \times 10^{5}$ \\
$X_{\text {Brij-30 }}$ & $a_{1}$ & $\tau_{1}(\mathrm{~ns})$ & $a_{1}$ & $\tau_{1}(\mathrm{~ns})$ & $\langle\tau\rangle(\mathrm{ns})$ & $\Delta v\left(\mathrm{~cm}^{-1}\right)$ & $\left(\mathrm{s}^{-\mathbf{1}}\right)$ \\
\hline 0 & 0.28 & 0.30 & 0.72 & 2.15 & 1.63 & 1900 & 1.99 \\
0.2 & 0.29 & 0.26 & 0.71 & 1.70 & 1.28 & 1880 & 2.26 \\
0.4 & 0.30 & 0.20 & 0.70 & 1.40 & 1.04 & 1870 & 3.29 \\
0.6 & 0.35 & 0.17 & 0.65 & 1.30 & 0.90 & 1840 & 5.06 \\
0.8 & 0.44 & 0.14 & 0.56 & 1.10 & 0.67 & 1800 & 7.27 \\
1.0 & 0.47 & 0.12 & 0.53 & 1.00 & 0.59 & 1760 & 12.3
\end{tabular}

curves with biexponential function. It could be observed from the table that the time constants are in the order of hundreds of picoseconds and a few nanoseconds. Such a slow relaxation of the entrapped water inside the RM has previously been argued to be due to the solvation of the probe by interfacially trapped water molecules. $1,2,46,47,65$ It could be noted that an ultrafast component of the solvent response, which is due to the relaxation of bulk-like water (of the order of a few picoseconds ${ }^{39,41,66}$ ) is not resolvable in our experimental setup. But since we are concerned with the effect of the change in interfacial composition on the solvation dynamics, such a loss would not significantly affect our conclusions. The change in the average time constant $\left\langle\tau_{\mathrm{s}}\right\rangle$ with $X_{\mathrm{Brij}-30}$ is plotted in Figure 4 . It could be observed that with an increase in $X_{\text {Brij-30 }},\left\langle\tau_{\mathrm{s}}\right\rangle$ gradually decreases, indicating that the nonionic interface is offering faster relaxation of the water molecules. It has been reported earlier that the 


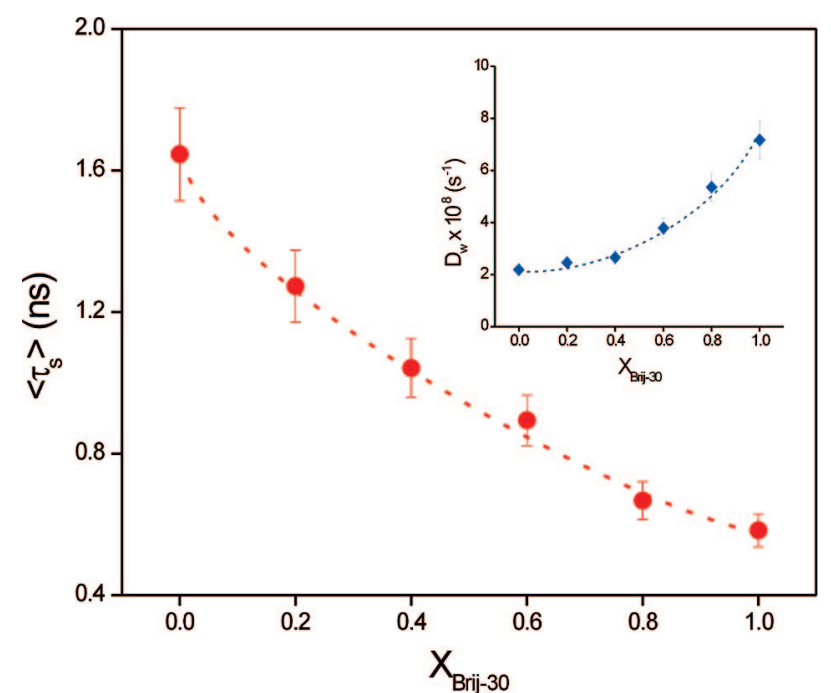

Figure 4. Change in $C(t)$ of AOT/Brij-30/isooctane/water mixed reverse micellar systems at $w_{0}=2.5$ as a function of $X_{\text {Brij-30. The }}$ corresponding diffusion coefficient values obtained from wobbling-incone analysis is shown in the inset. The broken lines are guide to the eye.

TABLE 2: Fluorescence Anisotropy Decays $(r(t))$ and Wobbling-in-Cone Data of C500 in Mixed RM at $X_{\mathrm{Brij}-30}{ }^{a}$

\begin{tabular}{lcccc}
\hline$X_{\text {Brij-30 }}$ & $r_{0}$ & $\tau_{\text {fast }}$ & $\tau_{\text {slow }}$ & $D_{W} \times 10^{8}\left(\mathrm{~s}^{-1}\right)$ \\
\hline 0 & 0.25 & $0.44(52 \%)$ & $2.70(48 \%)$ & 2.19 \\
0.2 & 0.24 & $0.43(58 \%)$ & $2.20(42 \%)$ & 2.46 \\
0.4 & 0.24 & $0.41(61 \%)$ & $1.90(39 \%)$ & 2.66 \\
0.6 & 0.24 & $0.27(55 \%)$ & $1.73(45 \%)$ & 3.79 \\
0.8 & 0.26 & $0.21(58 \%)$ & $1.50(42 \%)$ & 5.36 \\
1.0 & 0.26 & $0.17(62 \%)$ & $1.21(38 \%)$ & 7.17
\end{tabular}

${ }^{a}$ The figures in parentheses of the columns $\tau_{\text {fast }}$ and $\tau_{\text {slow }}$ signify the relative percentage of the components in the total anisotropy.

confinement of water molecules brings about the slow relaxation in RM irrespective of the charge type of the surface. ${ }^{38}$ However, it should also be considered that, depending upon the polar headgroup type and size, its interaction with water molecules might differ. In the present system, the nonionic surfactant Brij30 has four oxyethylene (EO) groups, each having different level of hydration. ${ }^{44}$ The present solvation result shows that the interaction of the EO groups with the water molecules is less strong than the corresponding AOT-RMs. Such an observation has previously been reported from the Levinger group using coumarin 153 as the probe. ${ }^{39}$

To ascertain the geometrical restriction of the probe in the interfacial region, we measure the time-resolved rotational anisotropy of the mixed surfactant systems. Typical anisotropy decay has been shown in the inset of Figure $3 \mathrm{~b}$. The decay transients have been fitted biexponentially and the results are shown in Table 2. It can be observed that the time constants are of the order of hundreds of picoseconds and a few nanoseconds, which is in the same order of magnitude as previously reported for AOT-RM systems. ${ }^{46,47,67}$ It could be noted that the $r_{0}$ values reported in the present systems are smaller than the ideal value of $0.4^{52}$ (Table 2). The limited resolution of our picosecond resolved experimental setup $(\sim 80$ ps IRF) is unable to detect the ultrafast components of the rotational motion, which eventually reduces the $r_{0}$ values. With an increase in $X_{\text {Brij-30, }}$, the time constants become faster indicating the probe experiencing less restriction at the interface. To understand the effect of $X_{\mathrm{Brij}-30}$ on the rotational relaxation process of the probe inside the RM water core in a more quantitative manner, the biexponential anisotropy decay has been analyzed using the two-step wobbling-in-cone model. ${ }^{68-70}$ Assuming that the slow and fast motion associated with the anisotropy decay are separable, the slow $\left(\tau_{\text {slow }}\right)$ and fast $\left(\tau_{\text {fast }}\right)$ rotational time constants can be related as,

$$
\begin{gathered}
\frac{1}{\tau_{\text {slow }}}=\frac{1}{\tau_{L}}+\frac{1}{\tau_{M}} \\
\frac{1}{\tau_{\text {fast }}}=\frac{1}{\tau_{W}}+\frac{1}{\tau_{\text {slow }}}
\end{gathered}
$$

where, $\tau_{L}$ and $\tau_{W}$ are the time constants for the lateral diffusion and the wobbling motion of the probe, respectively. $\tau_{M}$ is the time constant for the overall rotation of the RM and is given by the Stokes-Einstein-Debye equation

$$
\tau_{M}=\frac{\eta V_{h}}{k_{b} T}
$$

where $V_{h}$ is the hydrodynamic volume of the RM, $\eta$ is the viscosity of the dispersing solvent. It could be noted that $\tau_{M}$ values are order of magnitude higher than the $\tau_{\text {fast }}$ and $\tau_{\text {slow }}$ values. Hence, the overall rotation of the RM does not contribute to the decay of the anisotropy. In view of this, $\tau_{\text {fast }}$ and $\tau_{\text {slow }}$ essentially represent the time constants for wobbling motion and lateral diffusion respectively.

According to this model, the rotational anisotropy decay function is denoted as,

$$
r(t)=r_{0}\left[\beta \mathrm{e}^{-t / \tau_{\text {slow }}}+(1-\beta) \mathrm{e}^{-t / \tau_{\text {fast }}}\right]
$$

where $\beta=S^{2}$, and $S$ is the generalized order parameter that describes the degree of restriction on the wobbling-in-cone orientational motion. Its magnitude is considered as a measure of the spatial restriction of the probe and can have value from zero (for unrestricted rotation of the probe) to one (for completely restricted motion). The diffusion coefficient for wobbling motion $D_{W}$ can be obtained from the following relation,

$$
\begin{array}{r}
D_{W}=\frac{1}{\left(1-S^{2}\right) \tau_{W}}\left[\frac{x^{2}(1+x)^{2}}{2(x-1)}\left\{\ln \left(\frac{1+x}{2}\right)+\frac{1-x}{2}\right\}+\right. \\
\left.\frac{1-x}{24}\left(6+8 x-x^{2}-12 x^{3}-7 x^{4}\right)\right]
\end{array}
$$

where $x=\cos \theta_{W}$, the semicone angle given by, $S=1 / 2 \cos$ $\theta_{W}\left(1+\cos \theta_{W}\right)$. The calculated diffusion coefficients $\left(D_{W}\right)$ as a function of $X_{\mathrm{Brij}-30}$ are given in Table 2 and Figure 4 (inset). The obtained $D_{W}$ values are of the same order of magnitude as reported earlier for AOT-RM systems. ${ }^{47,67}$ It can be observed from Table 2 and Figure 4 that $D_{W}$ gradually increases with increasing $X_{\text {Brij-30 }}$ indicating that the probe experiences less restricted rotation in the $\mathrm{RM}$ and the microviscosity in the interfacial region decreases gradually. This might be due to the faster movement of the interfacial water at higher $X_{\text {Brij-30 }}$ values, once more indicating to a lesser interaction of the headgroup with water, confirming the previous solvation, FTIR and compressibility studies.

From the above discussions, it is evident that the change in the interfacial composition brings about change in the water dynamics for the AOT/Brij-30 mixed RM system. Now we question whether this change is reflected in the reaction property of the confined water molecules. To answer this we study the kinetics of a simple solvolysis reaction of benzoyl chloride $(\mathrm{BzCl})$. The reaction is a well studied one in the restricted medium ${ }^{71-73}$ and is reported to follow a simple first order kinetics. $\mathrm{BzCl}$ is solubilized in $i$-Oc 

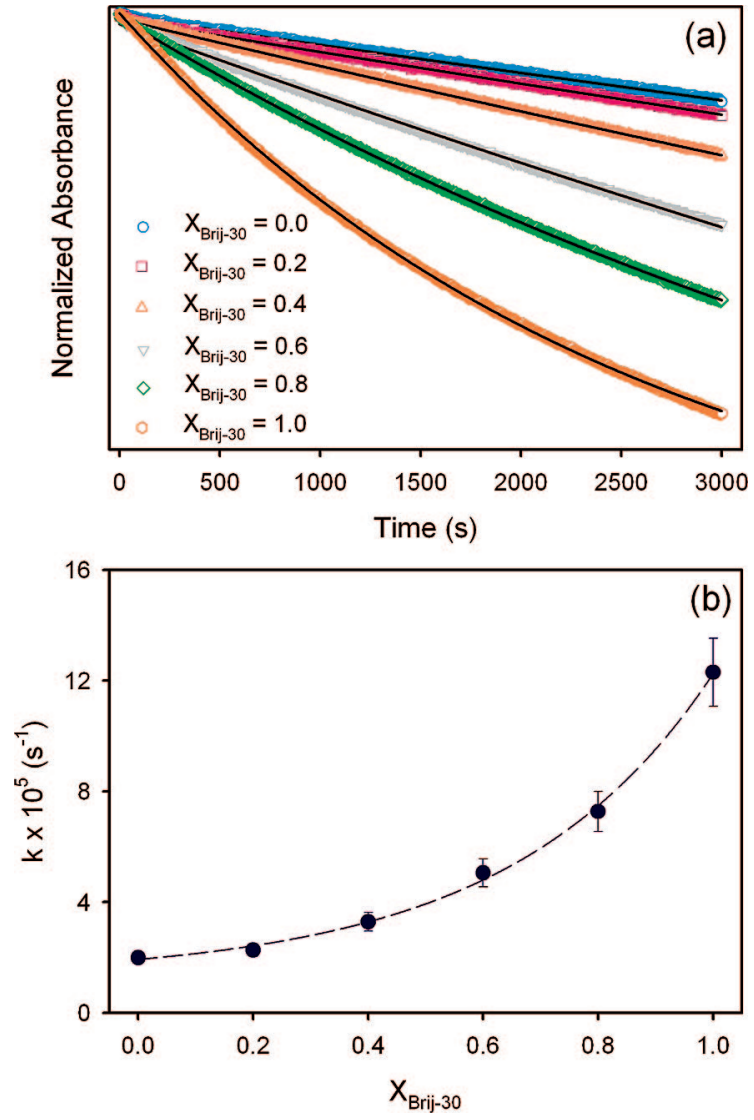

Figure 5. (a) Time dependent change on the absorbance of benzoyl chloride monitored at $288 \mathrm{~nm}$ for different mixed RM systems. The solid lines are fitted curves. (b) The rate of solvolysis (k) of benzoyl chloride in AOT/Brij-30/isooctane/water mixed reverse micellar systems at $\mathrm{w}_{0}=2.5$ as a function of $\mathrm{X}_{\mathrm{Brij}-30}$. The broken line is a guide to the eye.

and then added into the RM, wherein it gets compartmentalized into the organic phase and the interphase (with a partition coefficient of $K_{\mathrm{oi}}$ ). The interfacial water acts as a nucleophile to cleave the $\mathrm{C}-\mathrm{Cl}$ bond. The reaction can be monitored as the rate of decay of the absorbance of $\mathrm{BzCl}$ at $288 \mathrm{~nm}$. The decay kinetics of the mixed RM systems is shown in Figure 5. The corresponding observed rate constants $\left(k_{\mathrm{obs}}\right)$ of the reaction are given in Table 1 and are plotted against $X_{\mathrm{Brij}-30}$ in the same figure. The observed rate constants obtained for the single surfactant systems are comparable to the intrinsic rate constants obtained earlier, ${ }^{71,73}$ which are in turn much slower than that in pure water $\left(k=1.1 \mathrm{~s}^{-1}\right){ }^{73}$ It can be found that with increasing $X_{\mathrm{Brij}-30}$ the reaction kinetics becomes faster indicating that incorporation of the nonionic surfactants into the AOT interface not only increases the dynamics of the water molecules, it also makes a nucleuophilic reaction to occur with a faster rate constant. It has been reported earlier, ${ }^{71,73}$ that the rate of the solvolysis reaction increases as $w_{0}$ increases, which might be due to the increase in the amount of bulk-like water in the RM

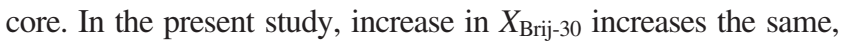
which in turn accelerates the reaction rate. Thus the faster dynamics of water molecules as evidenced from the solvation study, which is due to the weaker interaction of the nonionic surfactant with the water molecules as evidenced from FTIR and compressibility study, is manifested in the faster kinetics. It is to be noted that the rate constants reported in the present study are the observed rate constants $\left(k_{\mathrm{obs}}\right)$ and the change in the observed rate constants are rationalized as a function $\mathrm{X}_{\mathrm{Brij}-30}$. As mentioned earlier, the observed rate constant $\left(k_{\mathrm{obs}}\right)$ is a function of the partition constant of the reactant between the oil phase and interphase $\left(K_{\mathrm{oi}}\right) \cdot{ }^{71,73}$ To have a proper estimate of $K_{\mathrm{oi}}$ for different mixed systems, $k_{\mathrm{obs}}$ should be measured at different $\mathrm{w}_{0}$ values. In the present mixed systems, it is difficult to measure $k_{\mathrm{obs}}$ at different $w_{0}$ as there are nonisotropic regions in these systems at various $\mathrm{w}_{0}$ values (see Figure 1). However, the values of $K_{\mathrm{oi}}$ do not differ much for the pure AOT $\left(K_{\mathrm{oi}}=1.8\right.$ at $\left.w_{0}=3^{71}\right)$ and pure Brij-30 $\left(K_{\mathrm{oi}}=1.5\right.$ at $\left.w_{0}=2.5^{73}\right)$ RM systems, and it could be assumed that mixing of these two surfactants would not significantly affect the $\mathrm{K}_{\mathrm{oi}}$ values. In this respect our discussion on the effect of $X_{\mathrm{Brij}-30}$ on the reaction kinetics can be rationalized in the light of the $k_{\mathrm{obs}}$ values (Table 1).

The rate of solvolysis in the mixed surfactant system (Table 1)

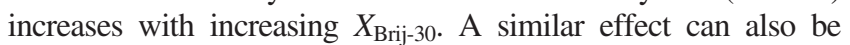
obtained by increasing the $w_{0}$ of single surfactant systems. Let us now compare the rate of hydrolysis in these two cases. As observed from the earlier report by Garcia Rio et al. ${ }^{71}$ the rate of solvolysis of $\mathrm{BzCl}$ in AOT-RM is dependent on $w_{0}$, but the dependency is quite complex in nature. From $w_{0}=2.0$ to 10.0 , the value of $k$ does not change significantly. But beyond $w_{0}=10$, it shows an exponential growth to saturate at $w_{0}=40$. It could be noted that the size of the waterpool of AOT-RM increases linearly with increasing $w_{0}$. Thus a 5-fold increase in $w_{0}$ (from $w_{0}=2$ to 10) proportionally increases the RM size and if the size of the RM is supposed to be the key factor to control the reaction rate, a proportionate increase in $k$ would also have been envisaged. Also the exponential increase in $k$ upon 4-fold increase in $w_{0}$ (from $w_{0}$ $=10$ to 40 ) could not be explained solely from the increase in size. In the present study, the rate of solvation shows a regular increasing trend with $X_{\mathrm{Brij}-30}$ at a fixed $w_{0}$ of 2.5 (Figure 5). A 1.7-fold increase in the size of the RM from $X_{\mathrm{Brij}-30}=0$ to 0.8 (Figure 2a) causes a 3.6-fold increase in $k$ (Table 1). Thus one can use the mixing of surfactants to modulate the nucleophilicity of the entrapped water in a more efficient manner than to increase the $\mathrm{w}_{0}$ of AOT-RM. It might be mentioned here that Brij-30 RMs are bigger in size than the AOT RMs (DLS study, Figure 2a) and the aggregation number of the former is much higher than the latter one for a fixed $\mathrm{w}_{0}$, which means that there are more water inside a single RM droplet for Brij-30 RM relative to the AOT RM. So, a manifold increase in the reaction rate of solvolysis of $\mathrm{BzCl}$ is expected for the former case, which indeed has been observed in the present study (Table 1). In case of mixed systems, as evidenced from the DLS study (Figure 2a), there is a systematic increase in the waterpool size with $X_{\text {Brij-30, which in turn indicates an increase }}$ in the aggregation number in the RM, and correspondingly the reaction rate increases (Table 1). The systematic increase in the droplet size (and also of the aggregation number) of the mixed $\mathrm{RM}$ with $X_{\mathrm{Brij}-30}$ is the key feature of the present study which in turn could be exploited to modulate the water structure, dynamics and reactivity. To generalize the effect of nonionic surfactant on the dynamics and functionality of confined water, an elaborate study involving nonionic surfactants of varying carbon chain length and polar headgroup size is needed, and such a study is underway in our laboratory.

\section{Conclusions}

Our studies explore the change in the dynamics of water entrapped in a RM core with the change in the composition of the interface by mixing of surfactants of different charge types. It is found that the hydrodynamic diameter of RM droplets suffers a small increase when $X_{\text {Brij-30 increases. FTIR and compressibility }}$ studies show that the entrapped water reaches more bulk-like behavior as $X_{\text {Brij-30 }}$ increases, which is due to the weaker interaction of Brij-30 headgroup with water compared to the charged headgroup of AOT. Solvation dynamics of C500 becomes faster with increasing $X_{\text {Brij-30 }}$. Rotational anisotropy study and wobbling-in- 
cone analysis confirms that the diffusion coefficient of water at the interface increases with $X_{\mathrm{Brij}-30}$ which is manifested in the faster solvation dynamics. Also the solvolysis of $\mathrm{BzCl}$ becomes faster with $X_{\text {Brij-30 }}$ indicating the increased nucleophilicity of the entrapped water caused by the faster diffusion of water molecules as evidenced from the solvation studies. Our study opens a new window to tune both the dynamics and reactivity of confined water molecules in RM only by altering the interfacial surfactant composition. This study will be helpful in further development of this microheterogeneous environment in the field of biomimicing science and technology.

Acknowledgment. We thank DST for a financial grant (SR/ FTP/PS-05/2004). P.K.V. thanks CSIR for a Research Fellowship.

Supporting Information Available: A table of compressibility measurements (Table S1). This information is available free of charge via the Internet at http://pubs.acs.org.

\section{References and Notes}

(1) Bhattacharyya, K. Acc. Chem. Res. 2003, 36, 95-101. 124

(2) Levinger, N. E. Curr. Opin. Colloid Interface Sci. 2000, 5, 118-

(3) Shinshi, M.; Sugihara, T.; Osakai, T.; Goto, M. Langmuir 2006, $22,5937-5944$

(4) Feng, X.; Zhang, J.; Chen, J.; Han, B.; Shen, D. Chem.-Eur. J. 2006, 12, 2087-2093.

(5) Krishna, S. H.; Srinivas, N. D.; Raghavarao, K. S. M. S.; Karanth, N. G. Adv. Bioch. Eng. Biotech. 2002, 75, 119-183.

(6) Melo, E. P.; Aires-Barros, M. R.; Cabral, J. M. S. Biotech. Ann. Rev. 2001, 7, 87-129.

(7) Leser, M. E.; Wei, G.; Luisi, P. L.; Maestro, M. Biochem. Biophys. Res. Commun. 1986, 135, 629-635. 4452 .

(8) Maruyama, T.; Hosogi, T.; Goto, M. Chem. Comm. 2007, 43, 4450-

(9) Streitner, N.; Voss, C.; Flaschel, E. J. Biotechnol. 2007, 131, 188196.

(10) Furusaki, S.; Ichikawa, S.; Goto, M. Prog. Biotechnol. 2000, 16 133-136. 193

(11) De, T. K.; Maitra, A. Adv. Colloid Interface Sci. 1995, 59, 95-

(12) Nave, S.; Eastoe, J.; Heenan, R. K.; Steytler, D.; Grillo, I. Langmuir 2000, 16, 8741-8748.

(13) Kunieda, H.; Shinoda, K. J. Colloid Interface Sci. 1985, 107, $107-$ 121.

(14) Hou, M. J.; Shah, D. O. Langmuir 1987, 3, 1086-1096.

(15) Huibers, P. D. T.; Shah, D. O. Langmuir 1997, 13, 5762-5765.

(16) Liu, D.; Ma, J.; Cheng, H.; Zhao, Z. Colloids Surf. A: Physicochem. Eng. Asp. 1998, 143, 59-68.

(17) Mitra, R. K.; Paul, B. K. Colloids Surf. A 2005, 255, 165-180.

(18) Paul, B. K.; Mitra, R. K. J. Colloid Interface Sci. 2005, 288, 261279.

(19) Seedher, N.; Manik, M. J. Surf. Sci. Technol. 1993, 9, 81-86.

(20) Liu, D.; Ma, J.; Cheng, H.; Zhao, Z. Colloids Surf. A: Physicochem Eng. Asp. 1998, 135, 157-164.

(21) Nazario, L. M. M.; Hatton, T. A.; Crespo, J. P. S. G. Langmuir 1996, 12, 6326-6335. 276.

(22) Bumajdad, A.; Eastoe, J. J. Colloid Interface Sci. 2004, 274, 268-

(23) Bumajdad, A.; Eastoe, J. Phys. Chem. Chem. Phys. 2004, 6, 15971602.

(24) Mitra, R. K.; Paul, B. K. Colloids Surfaces A: Physicochem. Eng. Asp. 2005, 252, 243-259.

(25) Kinugasa, T.; Kondo, A.; Nishimura, S.; Miyauchi, Y.; Nishii, Y.; Watanabe, K.; Takeuchi, H. Colloids Surf., A: Physicochem. Eng. Asp. 2002, 204, 193-199.

(26) Liu, D.; Ma, J.; Cheng, H.; Zhao, Z. Colloids Surf. A: Physicochem. Eng. Asp. 1998, 139, 21-26.

(27) Chatterjee, S.; Mitra, R. K.; Paul, B. K.; Bhattacharya, S. C. J. Colloid Interface Sci. 2006, 298, 935-941.

(28) Chatterjee, S.; Nandi, S.; Bhattacharya, S. C. Colloids Surf. A: Physicochem. Eng. Asp. 2006, 279, 58-63.

(29) Bumajdad, A.; Eastoe, J.; Heenan, R. K.; Lu, R. J.; Steytler, D. C. S.; Egelhaaf, S., E. J. Chem. Soc., Faraday Trans. 1998, 94, 2143 2150.

(30) Bumajdad, A.; Eastoe, J.; Griffiths, P.; Steytler, D. C.; Heenan, R. K.; Lu, J. R.; Timmins, P. Langmuir 1999, 15, 5271-5278.
(31) Bumajdad, A.; Eastoe, J.; Nave, S.; Steytler, D. C.; Heenan, R. K.; Grillo, I. Langmuir 2003, 19, 2560-2567.

(32) Li, Q.; Li, T.; Wu, J. J. Phys. Chem. B 2000, 104, 9011-9016.

(33) Shome, A.; Roy, S.; Das, P. Langmuir 2007, 23, 4130-4136.

(34) Chen, D.-H.; Liao, M.-H. J. Mol. Catal. B: Enzymatic 2002, 18 , $155-162$.

(35) Lan, J.; Zhang, Y.; Huang, X.; Hu, M.; Liu, W.; Li, Y.; Qu, Y.; Gao, P. J. Chem. Tech. Biotech. 2008, 83, 64-70.

(36) Poulsen, A. K.; Arleth, L.; Almdal, K.; Scharff-Poulsen, A. M. J. Colloid Interface Sci. 2007, 306, 143-153.

(37) Zhang, J.; Han, B.; Liu, J.; Zhang, X.; He, J.; Liu, Z.; Jiang, T.; Yang, G. Chem.-Eur. J. 2002, 8, 3879-3883.

(38) Moilanen, D. E.; Levinger, N. E.; Spry, D. B.; Fayer, M. D. J. Am. Chem. Soc. 2007, 129, 14311-14318.

(39) Pant, D.; Levinger, N. E. Langmuir 2000, 16, 10123-10130.

(40) Pant, D.; Riter, R. E.; Levinger, N. E. J. Chem. Phys. 1998, 109, 9995-10003.

(41) Riter, R. E.; Undiks, E. P.; Levinger, N. E. J. Am. Chem. Soc. 1998, 120, 6062-6067.

(42) Singh, P. K.; Satpati, A. K.; Kumbhakar, M.; Pal, H.; Nath, S. J. Phys. Chem. B 2008, ASAP.

(43) Vasilescu, M.; Caragheorgheopol, A.; Almgren, M.; Brown, W.; Alsins, J.; Johannsson, R. Langmuir 1995, 11, 2893-2898.

(44) Abel, S.; Waks, M.; Marchi, M.; Urbach, W. Langmuir 2006, 22, 9112-9120.

(45) Caldararu, H.; Caragheorgheopol, A.; Vasilescu, M.; Dragutan, I.; Lemmetyinen, H. J. Phys. Chem. 1994, 98, 5320-5331.

(46) Majumder, P.; Sarkar, R.; Shaw, A. K.; Chakraborty, A.; Pal, S. K. J. Colloid Interface Sci. 2005, 290, 462-474.

(47) Mitra, R. K.; Sinha, S. S.; Pal, S. K. Langmuir 2008, 24, 49-56.

(48) Zamyatnin, A. A. Аnnu. Rev. Biophys. Bioeng. 1984, 13, 145165.

(49) Sarvazyan, A. P. Annu. Rev. Biophys. Biophys. Chem. 1991, 20, $321-342$.

(50) Amararene, A.; Gindre, M.; Le Huerou, J.-Y.; Nicot, C.; Urbach, W.; Waks, M. J. Phys. Chem. B 1997, 101, 10751-10756.

(51) Mitra, R. K.; Sinha, S. S.; Pal, S. K. J.Phys.Chem.B 2007, 111, $7577-7581$.

(52) Lakowicz, J. R. Principles of fluorescence spectroscopy; Kluwer Academic/Plenum: New York, 1999.

(53) Mitra, R. K.; Paul, B. K. J. Colloid Interface Sci. 2005, 283, 565577.

(54) Evans, D. F.; Wennerström, H. The Colloidal Domain: Where Physics, Chemistry, Biology, and Technology Meet, 2nd ed.; Wiley-VCH: New York, 1999; p 560.

(55) Mukherjee, K.; Mukherjee, D. C.; Moulik, S. P. J. Colloid Interface Sci. 1997, 187, 327-333.

(56) Merdas, A.; Gindre, M.; Ober, R.; Nicot, C.; Urbach, W.; Waks, M. J. Phys. Chem. 1996, 100, 15180-15186.

(57) Leung, R.; Shah, D. O. J. Colloid Interface Sci. 1987, 120, 320329.

(58) Amararene, A.; Gindre, M.; Le Huérou, J.-Y.; Urbach, W.; Valdez, D.; Waks, M. Phys. Rev. E 2000, 61, 682-689.

(59) González-Blanco, C.; Rodríguez, L. J.; Velázquez, M. M. J. Colloid Interface Sci. 1999, 211, 380-386.

(60) Jain, T. K.; Varshney, M.; Maitra, A. J. Phys. Chem. 1989, 93, 7409-7416.

(61) Piletic, I. R.; Moilanen, D. E.; Levinger, N. E.; Fayer, M. D. J. Am. Chem. Soc. 2006, 128, 10366-10367.

(62) Piletic, I. R.; Moilanen, D. E.; Spry, D. B.; Levinger, N. E.; Fayer, M. D. J. Phys. Chem. A. 2006, 110, 4985-4999.

(63) Novaki, L. P.; Pires, P. A. R.; El Seoud, O. A. Colloid Polym. Sci. 2000, 178, 143-149.

(64) Zhong, Q.; Steinhurst, D. A.; Carpenter, E. E.; Owrutsky, J. C. Langmuir 2002, 18, 7401-7408.

(65) Sarkar, N.; Das, K.; Datta, A.; Das, S.; Bhattacharyya, K. J. Phys. Chem. 1996, 100, 10523-10527.

(66) Riter, R. E.; Willard, D. M.; Levinger, N. E. J. Phys. Chem. B. 1998, 102, 2705-2714.

(67) Shaw, A. K.; Pal, S. K. J. Phys. Chem. B 2007, 111, 4189-4199.

(68) Lipari, G.; Szabo, A. Biophys. J. 1980, 30, 489.

(69) Lipari, G.; Szabo, A. J. Chem. Phys. 1981, 75, 2971-2976.

(70) Lipari, G.; Szabo, A. J. Am. Chem. Soc. 1982, 104, 4546-4559.

(71) Garcia-Rio, L.; Leis, J. R.; Iglesias, E. J. Phys. Chem. 1995, 99 , $12318-12326$.

(72) Garcia-Rio, L.; Leis, J. R.; Mejuto, J. C. Langmuir 2003, 19, 31903197.

(73) Cabaleiro-Lago, C.; Garcia-Rio, L.; Herves, P.; Perez-Juste, J. J. Phys. Chem. B. 2005, 109, 22614-22622.

JP803585Q 